\title{
COMMENT OPEN Heterogeneity in clinical sequencing tests marketed for autism spectrum disorders
}

\author{
Ny Hoang ${ }^{1,2,3}$, Janet A. Buchanan ${ }^{4}$ and Stephen W. Scherer $2,3,4,5$ \\ npj Genomic Medicine (2018)3:27 ; doi:10.1038/s41525-018-0066-3
}

Autism spectrum disorders (ASD) is now a high profile and common concern in the population. Diagnosis has long been based on clinical findings, ${ }^{1}$ but with increased recognition of a strong genetic contribution and a subset of cases with an underlying genetic syndrome, ${ }^{2}$ clinical laboratory testing to search for genomic risk variants is now an important component of the diagnostic work-up. Chromosomal microarray is currently the recommended first-tier genetic test for $\mathrm{ASD}^{3}{ }^{3}$ revealing deleted and duplicated segments of DNA. These relatively large copy number variations (CNVs), often affecting several genes, can account for $5-25 \%$ of ASD cases, depending on the cohort examined. $^{4}$

The introduction of next-generation sequencing (NGS) now allows for a more in-depth look at ASD's genetic landscape. Besides CNVs, genomic risk factors for ASD can include singlenucleotide variants, insertion/deletions, and complex structural variations, contained in potentially hundreds of different genes, which may be involved in complex interactions. ${ }^{4-9}$ The NGS technology may be targeted to a selection of genes of interest, or to the coding portions of all genes (exome sequencing), or for entire genome sequencing. Such sequencing approaches are now being marketed for use as second-tier tests for ASD, particularly when chromosomal microarray analysis has not revealed any explanation for the clinical presentation.

We undertook to survey the scope of sequencing tests for ASD (or including ASD) that are primarily being marketed by commercial laboratories as adjuncts or follow-up to chromosomal microarrays. Perhaps not surprisingly, because this is a new territory and guidelines are not yet developed, we found significant heterogeneity among such laboratories with respect to the tests they offer. The most striking finding was the variable number of genes being tested for on panels marketed for ASD (range, 11-2562), with little content overlap, albeit, these encompassed ASD-specific as well as much larger ASD-inclusive panels (Table 1 ).

Our search began in 2017, using the former GeneTests and current Genetic Testing Registry as resources, supplemented by an internet search (terms: "autism panel", "autism lab sequencing", "autism genetic test"). To focus on gene panels, we excluded biochemical assays, chromosomal microarrays, and sequencing tests involving fewer than three genes. To focus on ASD, we excluded tests targeted for general neurodevelopmental disorders, seizure disorders, and intellectual disability, unless they specified autism. That search resulted in 20 DNA testing laboratories offering an ASD gene panel. Updating the survey in June 2018 (adding use of a new resource, Concert Geneticswww.concertgenetics.com), we found that four panels were no longer offered, but five were newly available, for an updated total of 21 laboratories (Table 1). We then went to the individual laboratory websites for further information.

We compared ASD gene lists from panels of the 21 laboratories. Each entire panel is listed in Supplementary Table S1. Supplementary Table S2 shows the 178 genes included in at least five lab panels ("shared genes") in order of listing frequency. Table 2 summarizes the top 16 most commonly listed genes, along with their associated genetic disorders according to the Online Mendelian Inheritance in Man (OMIM). ${ }^{10}$ The latter shows that most (12/16) of these genes are associated with genetic syndromes, where the primary phenotype involves physical/ systemic features and not ASD. Almost half of these genes (7/ 16) were located on the $X$ chromosome. Only one gene was included on all panels offered by the 21 labs: $M E C P 2$, which is associated with Rett syndrome (previously considered part of the ASD spectrum, but no longer so under criteria of the Diagnostic and statistical manual of mental disorders (5th edn)). ${ }^{1}$ There were 63 genes shared among at least 10 lists, but the vast majority of the cumulative list of 2928 unique genes were included by fewer than five laboratories. There were, nonetheless, some pockets of significant overlap. Two pairs of laboratories each posted identical ASD gene lists. Two other laboratories had lists that encompassed those of separate labs, but with additional genes to create their own collection.

We then compared the gene lists ascertained here to four lists from academic research projects that identify genes with strong association to ASD: Simons Foundation Autism Risk Initiative (SFARI), ${ }^{11}$ Simons Foundation Powering Autism Research for Knowledge (SPARK), ${ }^{12}$ Autism Speaks - MSSNG, ${ }^{8}$ and Autism Sequencing Consortium (ASC) ${ }^{13}$ (Supplementary Table S2). Comparing these lists, we found 15 genes to be shared by all, and an additional 24 genes were common to 3 of the 4 lists (Supplementary Table S3). We noted that 39 ASD risk genes identified by at least two of these research sources were not included among the top 178 genes listed by the commercial labs (Supplementary Table S4). Moreover, seven well-studied genes (ADNP, ARID1B, CHD8, POGZ, SCN2A, SLC6A1, and SYNGAP1) identified to be important for ASD by the SFARI, SPARK, MSSNG, and ASC research projects, are not found among the commercial list of the top 47 shared genes (Supplementary Table S2).

\footnotetext{
${ }^{1}$ Department of Genetic Counselling, The Hospital for Sick Children, Toronto, ON, Canada; ${ }^{2}$ Genetics and Genome Biology, The Hospital for Sick Children, Toronto, ON, Canada;

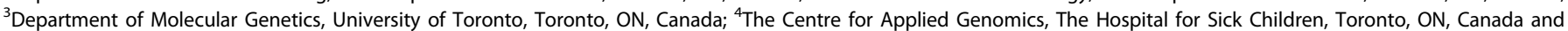
${ }^{5}$ McLaughlin Centre and Department of Molecular Genetics, Faculty of Medicine, University of Toronto, Toronto, ON, Canada Correspondence: Ny Hoang (ny.hoang@sickkids.ca) or Stephen W. Scherer (stephen.scherer@sickkids.ca)
}

Received: 1 August 2018 Revised: 23 August 2018 Accepted: 29 August 2018

Published online: 19 September 2018 


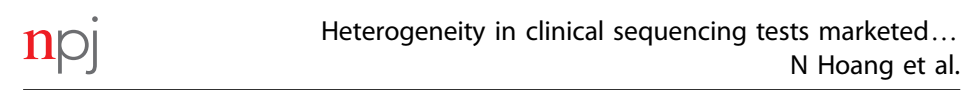

2

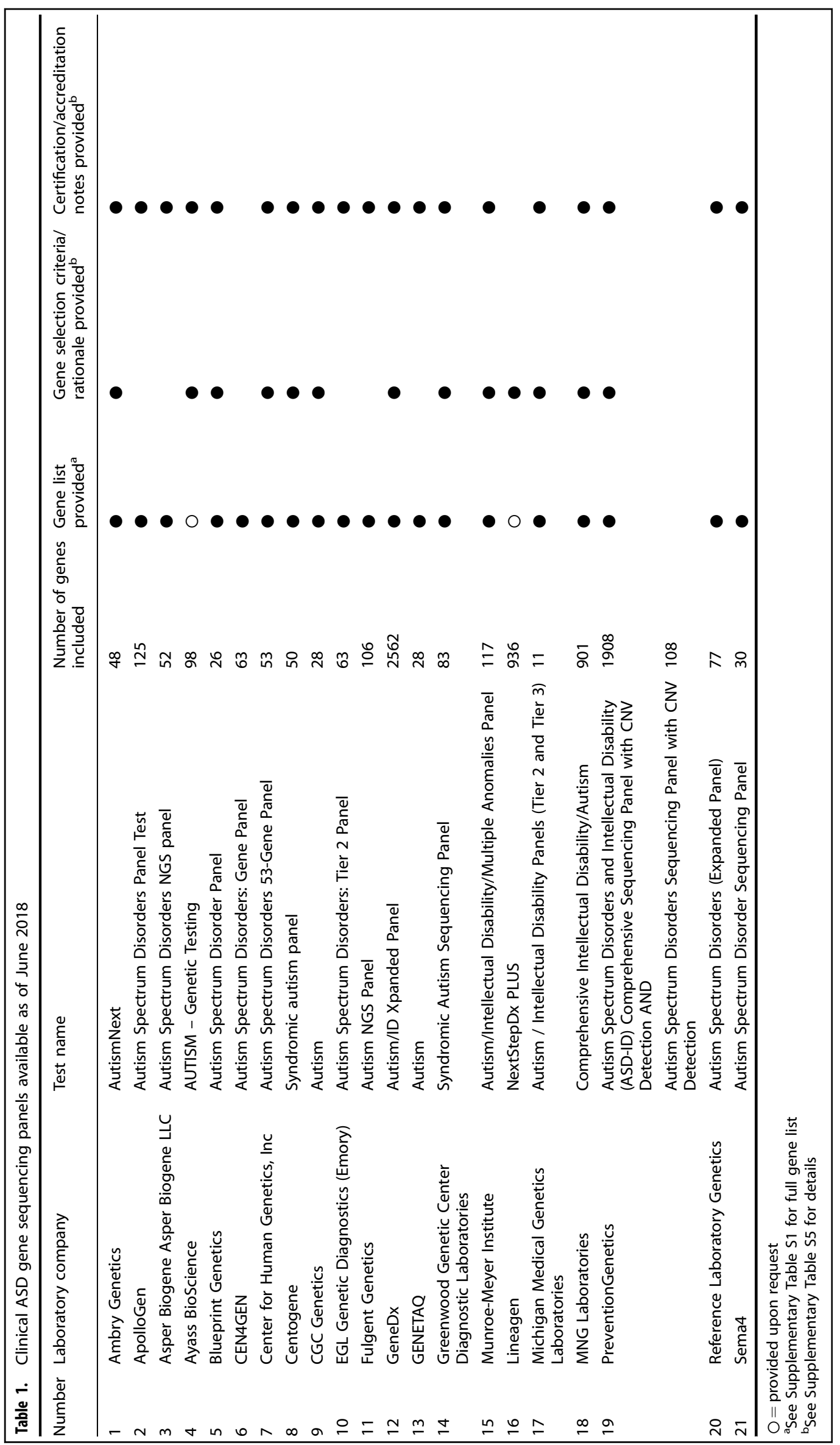


Table 2. Summary of findings from gene list comparison and list of most commonly listed genes

Key findings

- Number of genes included on ASD gene sequencing panels ranged from 11 to 2562

2928 unique genes identified across 21 labs

When comparing the gene lists of 21 labs (see Supplementary Table S1)

O Only one gene (MECP2) was shared by all labs

O 63 genes shared by at least 10/21 labs

OTwo sets of labs had the same gene lists

- Of the top 16 genes shared by 16 or more panels,

O 12 were associated with known OMIM syndromes ${ }^{\text {a }}$ primarily involving physical/systemic features, of which 3 (MECP2, PTEN, and CNTNAP2) included autism susceptibility

O 4 (NLGN4X, PTCHD1, NLGN3, and FOXP1) were associated with primarily a neurocognitive presentation (ASD, intellectual disability) in OMIM

O 2 (UBE3A and SHANK3) overlapped with known ASD-associated CNV regions

- The top 178 genes shared across 21 labs did NOT include 39 genes cited on 2 or more ASD gene lists ${ }^{c}$

Top shared genes ${ }^{b}$

Gene name Labs OMIM gene association (OMIM\#)

MECP2 ${ }^{C} \quad 21 / 21$ Encephalopathy (300673); Mental retardation (300260)(300055); Rett syndrome (312750); Autism susceptibility (300496)

NLGN4X 20/21 Mental retardation (300495); Asperger syndrome susceptibility (300497); Autism susceptibility (300495)

CACNA1C 19/21 Brugada syndrome 3 (611875); Timothy syndrome (601005)

NRXN1 ${ }^{\mathrm{C}} \quad 19 / 21$ Pitt-Hopkins-like syndrome 2 (614325); Schizophrenia susceptibility (614332)

PCDH19 19/21 Epileptic encephalopathy (300088)

PTCHD1 $1^{\mathrm{C}} \quad$ 19/21 Autism susceptibility (300830)

UBE3A 19/21 Angelman syndrome (105830)

NLGN3 ${ }^{c} \quad 18 / 21$ Asperger syndrome susceptibility (300494); Autism susceptibility (300425)

PTEN $\quad$ 18/21 Bannayan-Riley-Ruvalcaba syndrome (153480); Cowden syndrome 1/ Lhermitte-Duclos syndrome (158350); Macrocephaly/ autism syndrome (605309); VATER association with macrocephaly and ventriculomegaly (276950); Glioma susceptibility (613028); Meningioma (607174); Prostate cancer (176807)

SHANK3 ${ }^{\mathrm{C}} \quad$ 18/21 Phelan-McDermid syndrome (606232); Schizophrenia (613950)

CDKL5 ${ }^{c} \quad 17 / 21$ Epileptic encephalopathy (300672)

CNTNAP2 17/21 Cortical dysplasia-focal epilepsy syndrome/ Pitt-Hopkins like syndrome 1 (610042); Autism susceptibility (612100)

DHCRT $\quad 17 / 21$ Smith-Lemli-Opitz syndrome (270400)

FOXP1 $^{\mathrm{C}} \quad 17 / 21$ Mental retardation with language impairment and with or without autistic features (613670)

NSD $1^{\mathrm{c}} \quad 17 / 21$ Leukemia (601626); Sotos syndrome 1 (117550)

ARX $\quad 16 / 21$ Epileptic encephalopathy (308350); Hydranencephaly with abnormal genitalia (300215); Lissencephaly (300215); Mental retardation (300419); Partington syndrome (309510); Proud syndrome (300004)

${ }^{a}$ Online Mendelian Inheritance in Man (OMIM) is a continuously updated catalog of human genes and genetic disorders and traits, with particular focus on the molecular relationship between genetic variation and phenotypic expression. omim.org

${ }^{\mathrm{b}}$ List of the top 16 genes shared by 16 or more clinical ASD sequencing panels

${ }^{\mathrm{C}}$ Gene listed on 2 or more of the following: SFARI list ${ }^{11}$ with gene score of 1 or 2 ; SPARK list ${ }^{12}$ as of April 2017; MSSNG list published in $2017 ;^{8}$ ASC list ${ }^{13}$ of genes

with $q$ value $<0.3$

How were these lists derived by the 21 clinical laboratories? We looked for such information from the websites, documented the mention of gene selection criteria or rationale (Table 1) and summarized the comments found (Supplementary Table S5). Some labs indicated nothing, some made general comments, some specified a filter of "syndromic autism", and a few provided notes with or without literature citations. Favorably, one lab provided a dated record of updates to their panel.

From the respective websites, information about sequencing and analysis approaches used by the various laboratories were rarely obvious and sometimes unstated. A few of the labs undertook exome sequencing followed by selective analysis of an ASD gene panel. A few other labs stated that they applied NGS to "selected" or "targeted" genes only, and most did not provide any specification. Some added mention of exons or coding sequences; some also mentioned introns, splice junctions, or noncoding sequence. One lab clearly said they used Sanger sequencing only, and some noted that adjunct Sanger sequencing was applied either for regions poorly covered with NGS or for confirmation of significant findings before reporting.

Our ascertainment was intended to identify clinical tests; therefore, we searched for documentation of laboratory qualification and tabulated key findings (Table 1 and Supplementary Table S5). Additionally, a few websites specified that tests were not direct-to-consumer or could only be ordered by a health professional. Several labs mentioned that the test was intended for individuals with symptoms or clinical diagnosis of ASD, some limiting this to "syndromic"; few mentioned "pre-symptomatic", "prenatal", or "carrier" testing. Some described in-house genetic counseling support and/or the qualifications of those preparing reports. Few described their variant reporting protocol, some mentioning the classification scheme of the American College of Medical Genetics and Genomics. ${ }^{14}$

The diversity unearthed by this survey applied not only to the specific gene tests offered for ASD, but to the extent of supporting information provided by the companies who market these services. Consumers would benefit from greater transparency (including evidence) about items such as gene inclusion criteria, dates of updates, technologies used, interpretation of variants, reporting standards, ownership of data, secondary use policies, etc.

ASD is clearly a heterogeneous disorder, both in clinical presentation and in terms of the underlying etiology. ${ }^{15}$ In fact, the genetic predisposition for ASD may be different in almost 
every individual. ${ }^{6,9}$ Furthermore, the evidence for association between any given variant and ASD is wide-ranging, and even when an association is strongly supported, there is usually variable expressivity or some degree of non-penetrance reflected in the evidence. ${ }^{12,15}$ The NGS tests currently marketed for ASD are mostly being used in a confirmatory manner, in the context of clinical findings. However, expectations are rising, from families and their healthcare providers, to use the same DNA tests to enable early diagnosis, prognosis, and medical management of ASD, and to assess familial risk. This survey highlights the need to develop a clinically validated list of genes appropriate for clinical laboratory analysis for ASD to meet these growing demands.

\section{ACKNOWLEDGEMENTS}

The authors thank Jennifer Howe and R. Spencer Tong for assisting in database searches and assembling results. The research was supported with funds from the University of Toronto McLaughlin Centre, The Centre for Applied Genomics technology platform grant from Genome Canada, the Canadian Institutes of Health Research (CIHR) Foundation grant, the SickKids Foundation, and the GlaxoSmithKlineCIHR Endowed Chair in Genome Sciences held by S.W.S.

\section{AUTHOR CONTRIBUTIONS}

All authors conceptualized, researched, and wrote this paper.

\section{ADDITIONAL INFORMATION}

Supplementary information accompanies the paper on the npj Genomic Medicine website (https://doi.org/10.1038/s41525-018-0066-3).

Competing interests: S.W.S. and SickKids Hospital has licensed intellectual property for genetic testing in autism to Athena Diagnostics, Lineagen, Population Bio. S.W.S. is also on the Scientific Advisory Committees of Population Bio and Deep Genomics, and directs the Autism Speaks MSSNG Open Science project. The remaining authors declare no competing interests.

Publisher's note: Springer Nature remains neutral with regard to jurisdictional claims in published maps and institutional affiliations.

\section{REFERENCES}

1. American Psychiatric Association. Diagnostic and Statistical Manual of Mental Disorders 5th edn (American Psychiatric Publishing, Arlington, VA, 2013).

2. Fernandez, B. A. \& Scherer, S. W. Syndromic autism spectrum disorders: moving from a clinically defined to a molecularly defined approach. Dialog. Clin. Neurosci. 19, 353-371 (2017).
3. Miller, D. T. et al. Consensus statement: chromosomal microarray is a first-tier clinical diagnostic test for individuals with developmental disabilities or congenital anomalies. Am. J. Hum. Genet. 86, 749-764 (2010).

4. Tammimies, K. et al. Molecular diagnostic yield of chromosomal microarray analysis and whole-exome sequencing in children with autism spectrum disorder. JAMA 1, 314, 895-903 (2015).

5. De Rubeis, S. et al. Synaptic, transcriptional and chromatin genes disrupted in autism. Nature 13, 515, 209-15 (2014).

6. Yuen, R. K. et al. Whole-genome sequencing of quartet families with autism spectrum disorder. Nat. Med. 21, 185-191 (2015).

7. Sanders, S. et al. Insights into autism spectrum disorder genomic architecture and biology from 71 risk loci. Neuron 23, 87, 1215-1233 (2015).

8. Yuen, R. K. et al. Whole genome sequencing resource identifies 18 new candidate genes for autism spectrum disorder. Nat. Neurosci. 20, 602-611 (2017).

9. W einer, D. J. et al. Polygenic transmission disequilibrium confirms that common and rare variation act additively to create risk for autism spectrum disorders. Nat. Genet. 49, 978-985 (2017).

10. Online Mendelian Inheritance in Man, $\mathrm{OMIM}^{\oplus}$. McKusick-Nathans Institute of Genetic Medicine https://omim.org/ (Johns Hopkins University, Baltimore, MD, 2018).

11. Abrahams, B. S. et al. SFARI Gene 2.0: a community-driven knowledgebase for the autism spectrum disorders (ASDs). Mol. Autism 4, 36 (2013).

12. Feliciano, P. et al. SPARK: a US cohort of 50,000 families to accelerate autism research. Neuron 97, 488-493 (2018).

13. Larsen, E. et al. A systematic variant annotation approach for ranking genes associated with autism spectrum disorders. Mol. Autism 7, 44 (2016).

14. Richards, S. et al. Standards and guidelines for the interpretation of sequence variants: a joint consensus recommendation of the American College of Medical Genetics and Genomics and the Association for Molecular Pathology. Genet. Med. 17, 405-424 (2015).

15. Hoang, N., Cytrynbaum, C. \& Scherer, S. W. Communicating complex genomic information: a counselling approach derived from research experience with Autism Spectrum Disorder. Patient Educ. Couns. 101, 352-361 (2017).

Open Access This article is licensed under a Creative Commons Attribution 4.0 International License, which permits use, sharing, adaptation, distribution and reproduction in any medium or format, as long as you give appropriate credit to the original author(s) and the source, provide a link to the Creative Commons license, and indicate if changes were made. The images or other third party material in this article are included in the article's Creative Commons license, unless indicated otherwise in a credit line to the material. If material is not included in the article's Creative Commons license and your intended use is not permitted by statutory regulation or exceeds the permitted use, you will need to obtain permission directly from the copyright holder. To view a copy of this license, visit http://creativecommons. org/licenses/by/4.0/.

(c) The Author(s) 2018 